\title{
Contents
}

Part I Dark Matter: The Missing Matter of the Universe as Seen by Astroparticle Physics and Astrophysics

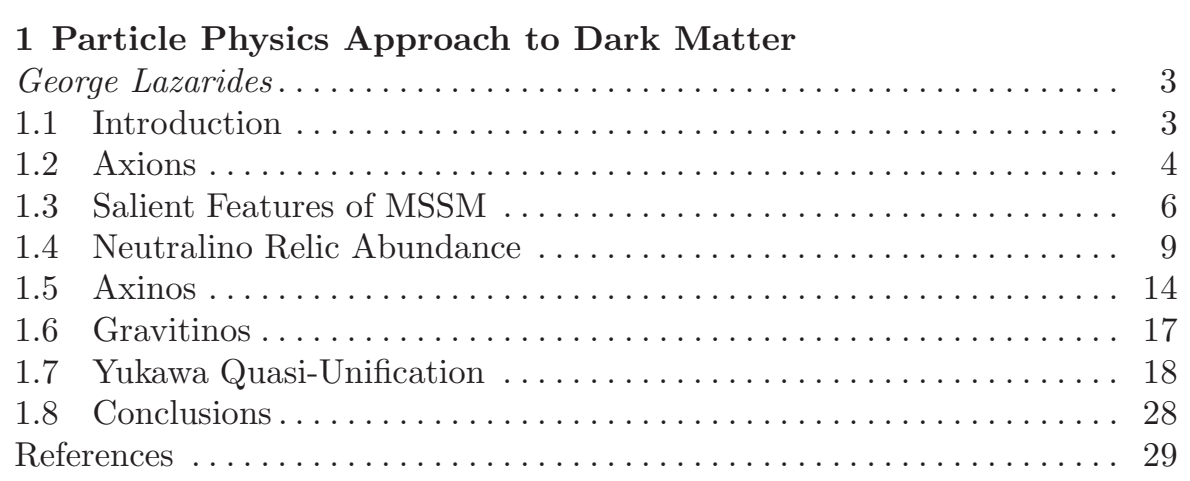

2 LSP as a Candidate for Dark Matter

Athanasios Lahanas ..................................... 35

2.1 Introduction .................................. 35

2.2 The Energy - Matter Content of the Universe $\ldots \ldots \ldots \ldots \ldots \ldots 37$

2.3 The Thermal Universe $\ldots \ldots \ldots \ldots \ldots \ldots \ldots \ldots \ldots \ldots \ldots . \ldots \ldots$

2.4 Dark Matter ................................. 46

2.5 Calculating DM Relic Abundances . ..................... 48

2.6 Supersvmmetry and its Cosmological Implications . . . . . . . . . . . 54

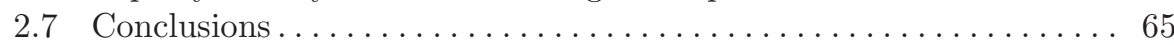

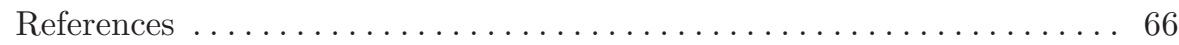

\section{On the Direct Detection of Dark Matter}

John Verqados ..................................... 69

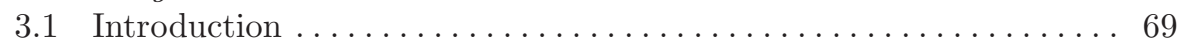

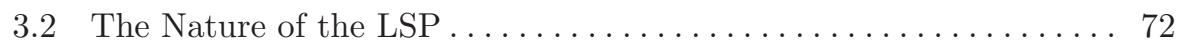

3.3 The Feynman Diagrams Entering the Direct Detection of LSP $\ldots 72$ 
3.4 Going from the Quark to the Nucleon Level . . . . . . . . . . . . . . . . . . 80

3.5 The Nucleon Cross Sections . . . . . . . . . . . . . . . . . . . . 84

3.6 The Allowed SUSY Parameter Space . . . . . . . . . . . . . . . . . . . . . . . . . . 84

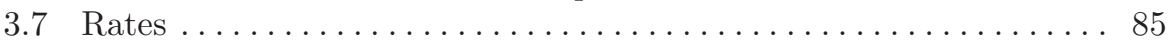

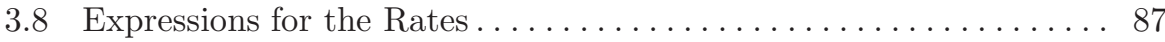

3.9 Bounds on the Scalar Proton Cross Section . . . . . . . . . . . . . . 89

3.10 Exclusion Plots in the $a_{n}, a_{n}$ and $\sigma_{p}, \sigma_{n}$ Planes $\ldots \ldots \ldots \ldots \ldots \ldots 90$

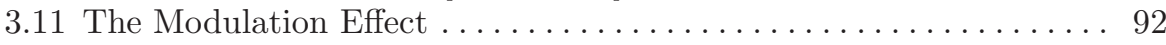

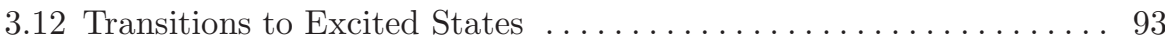

3.13 The Directional Rates . . . . . . . . . . . . . . . . . . . . 93

3.14 Observation of Electrons Produced During

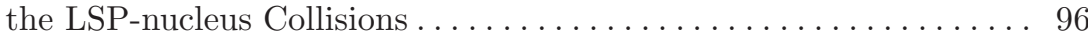

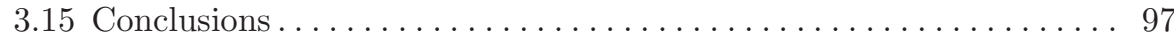

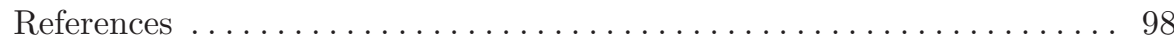

\section{Galaxy Formation and Dark Matter}

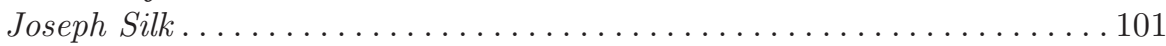

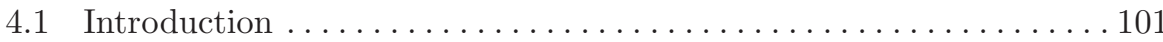

4.2 Precision Cosmology . . . . . . . . . . . . . . . . . . . . . . . . . . . . . . . . 102

4.3 The Global Baryon Inventory . . . . . . . . . . . . . . . . . . . . . . . . 104

4.4 The "Missing" Barvons . . . . . . . . . . . . . . . . . . . . . . . . . . . 105

4.5 Large-scale Structure and Cold Dark Matter: The Issues . . . . . . . . . . 106

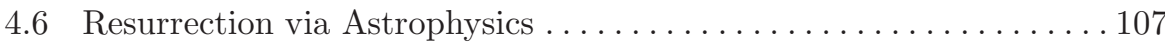

4.7 What Determines the Mass of a Galaxy? . . . . . . . . . . . . . . . . . 109

4.8 Disk Galaxy Formation . . . . . . . . . . . . . . . . . . . . . . . . . . 109

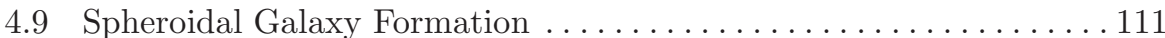

4.10 Numerical Simulations . . . . . . . . . . . . . . . . . . . . . . . 112

4.11 The Case for Positive Feedback . . . . . . . . . . . . . . . . . . . . . . . 115

4.12 Observing Cold Dark Matter: Where Next? . . . . . . . . . . . . . . . . . . 117

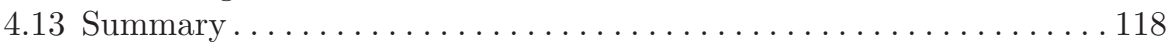

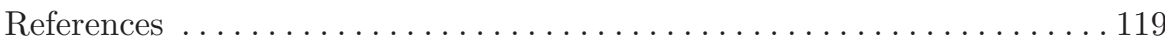

\section{Part II Dark Energy: The Energy Balance of the Universe} within the Standard Cosmological Model

\section{Cosmological Parameters from Galaxy Clusters:}

\section{An Introduction}

Paolo Tozzd .......................................... 125

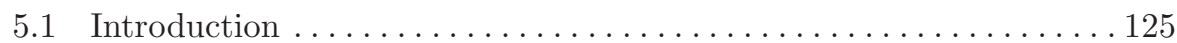

5.2 Clusters of Galaxies in a Cosmological Context . . . . . . . . . . . . . 126

5.3 From Observations to Cosmological Parameters . . . . . . . . . . . . . . . . 137

5.4 New Physics and Future Prospects. . . . . . . . . . . . . . . . . . . . 149

5.5 What to Bring Home . . . . . . . . . . . . . . . . . . . . . . 154

References ...................................... 155 


\section{Cosmological Constraints from Galaxy Clustering}

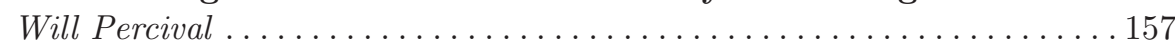

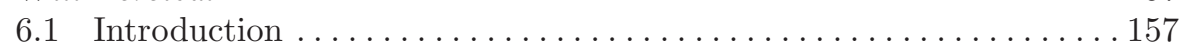

6.2 Basics . . . . . . . . . . . . . . . . . . . . . . . . 158

6.3 Matter Perturbations . . . . . . . . . . . . . . . . . . . . . . 159

6.4 The Evolution of Perturbations $\ldots \ldots \ldots \ldots \ldots \ldots \ldots \ldots \ldots \ldots \ldots \ldots \ldots$

6.5 Galaxy Survey Analysis . . . . . . . . . . . . . . . . . . . . 165

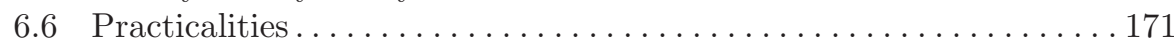

6.7 Results from Recent Survevs . . . . . . . . . . . . . . . . . . . 174

6.8 Combination with CMB Data . . . . . . . . . . . . . . . . . . 175

References ................................... 184

\section{Dark Energy and the Microwave Background}

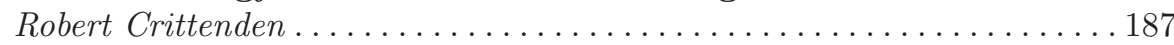

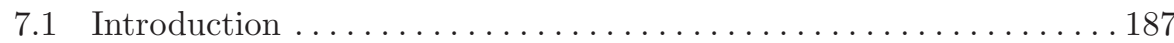

7.2 Models for Dark Energy . . . . . . . . . . . . . . . . . . . . . . . 189

7.3 The Physics of the Microwave Background . . . . . . . . . . . . . . . 192

7.4 Wavs of Probing Dark Energy . . . . . . . . . . . . . . . . . . . . . . . . . . . . 204

7.5 The Integrated Sachs-Wolfe Effect . . . . . . . . . . . . . . . . . . . . . . . . . . . . . . . . . 209

7.6 Conclusions and Future Prospects . . . . . . . . . . . . . . . . 212

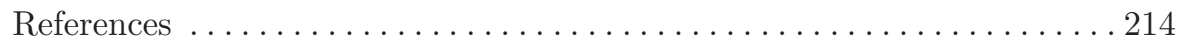

\section{Models of Dark Energy}

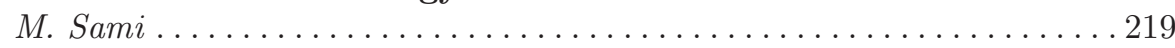

8.1 Glimpses of FRW Cosmology . . . . . . . . . . . . . . . . . . . . 220

8.2 Cosmological Constant 1 . . . . . . . . . . . . . . . . . . 2 236

8.3 Dvnamically Evolving Scalar Field Models of Dark Energy . . . . . . . 239

8.4 Scaling Solutions in Models of Coupled Quintessence . . . . . . . . . . . . 248

8.5 Quintessential Inflation . . . . . . . . . . . . . . . . . . . . . . 249

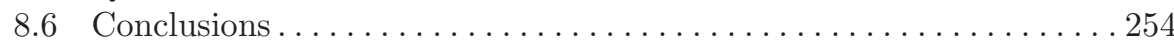

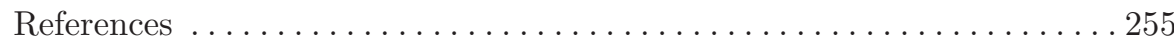

\section{Accelerating Universe: Observational Status}

\section{and Theoretical Implications}

Leandros Perivolaropoulos . ..............................257

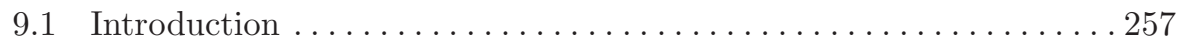

9.2 Expansion History from the Luminosity Distances of SnIa . . . . . . 258

9.3 Observational Results ................................ 265

9.4 Dark Energy and Negative Pressure $\ldots \ldots \ldots \ldots \ldots \ldots \ldots \ldots \ldots$

9.5 Dynamical Evolution of Dark Energy . . . . . . . . . . . . . . . . 2 278

9.6 The Fate of a Phantom Dominated Universe: Big Rip . . . . . . . . . 282

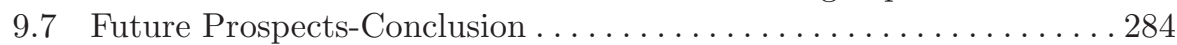

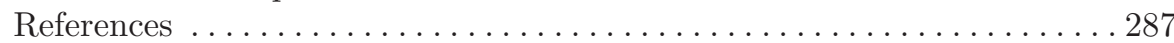


Part III Dark Matter and Dark Energy Beyond the Standard Theory of General Relativity

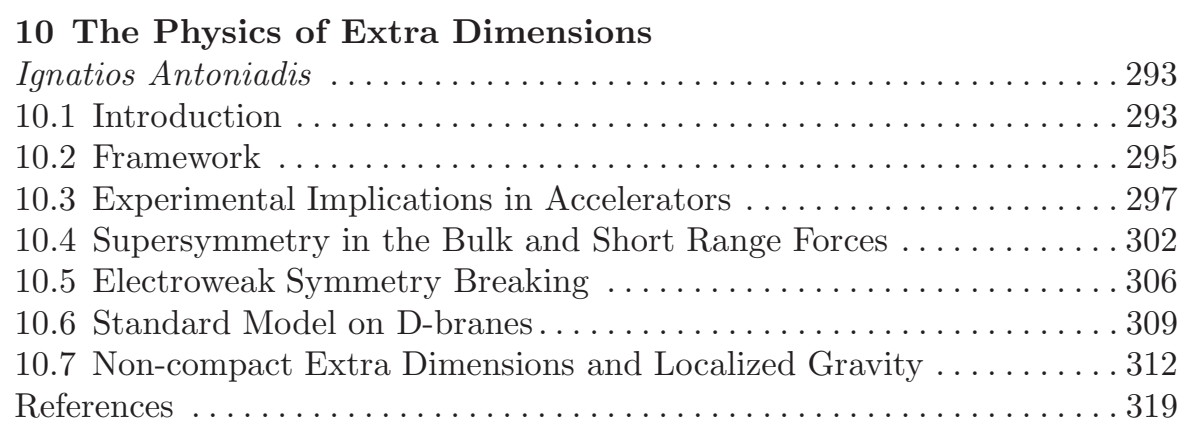

\section{Dark Energy from Brane-world Gravity}

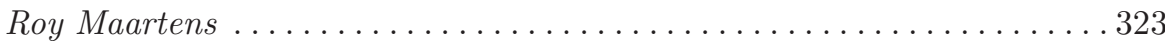

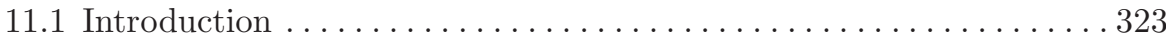

11.2 KK Modes of the Graviton . . . . . . . . . . . . . . . . . . . . . . . 326

11.3 DGP Type Brane-worlds: Self-accelerating Cosmologies . . . . . . . . 327

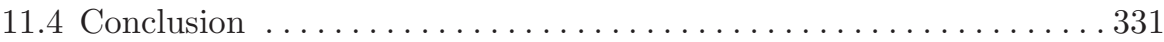

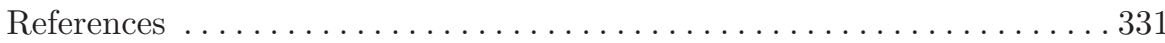

12 The Issue of Dark Energy in String Theory

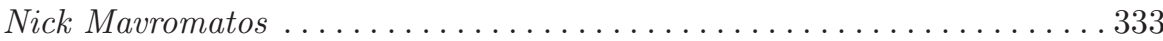

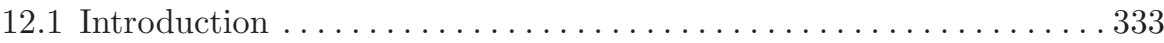

12.2 De Sitter $(\mathrm{dS})$ Universes from a Modern Perspective . . . . . . . . . . . 336

12.3 No Horizons in Perturbative (Critical) String Theory . . . . . . . . . . 343

12.4 Dilaton Quintessence in String Theory . . . . . . . . . . . . . . . . . . . . . 346

12.5 Conclusions . . . . . . . . . . . . . . . . . . . . . . . . . . 369

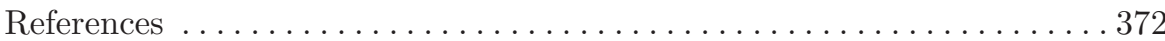

\section{Modified Gravity Without Dark Matter}

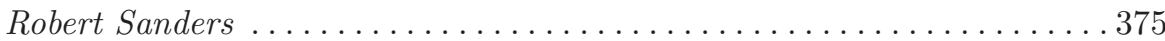

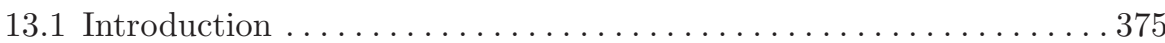

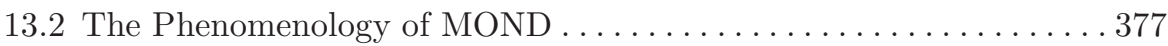

13.3 Relativistic MOND . . . . . . . . . . . . . . . . . . . . . . . . . . 385

13.4 TeVeS: Successes. Issues and Modifications . . . . . . . . . . . . . . 393

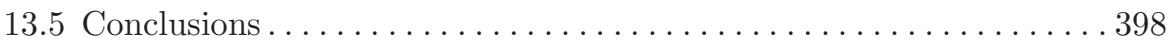

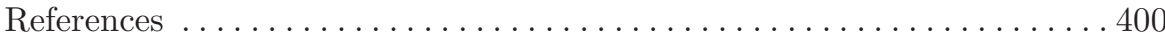

\section{Avoiding Dark Energy with 1/R Modifications of Gravity}

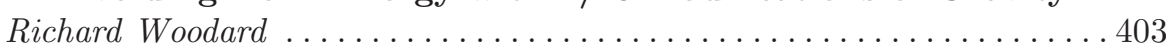

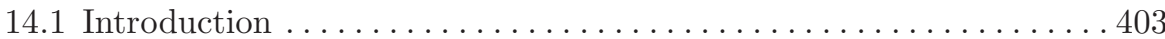

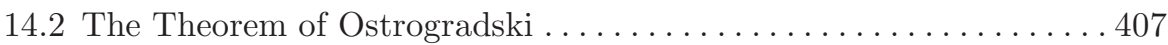

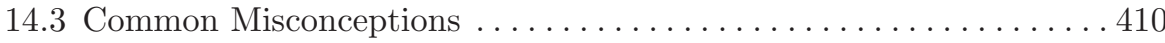




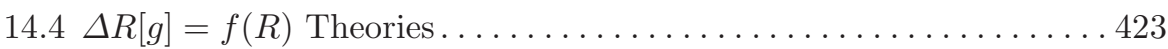

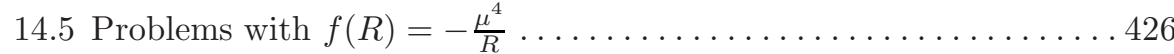

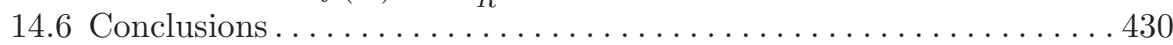

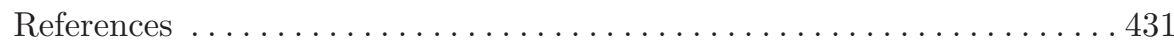

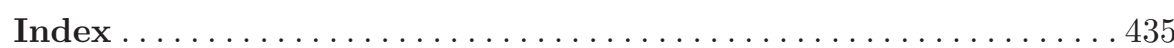

